\title{
Incidence of pulmonary embolism in non-critically ill COVID-19 patients. Predicting factors for a challenging diagnosis
}

\author{
B. Mestre-Gómez ${ }^{1}$ - R. M. Lorente-Ramos ${ }^{2}$. J. Rogado ${ }^{3}$ - A. Franco-Moreno ${ }^{1}$ - B. Obispo ${ }^{3}$ - D. Salazar-Chiriboga ${ }^{2}$. \\ T. Saez-Vaquero ${ }^{1}$. J. Torres-Macho ${ }^{1,9} \cdot$ A. Abad-Motos ${ }^{4}$. C. Cortina-Camarero ${ }^{5}$. A. Such-Diaz ${ }^{6}$. E. Ruiz-Velasco ${ }^{7}$. \\ J. Churruca-Sarasqueta ${ }^{8} \cdot$ N. Muñoz-Rivas ${ }^{1}$ on behalf of Infanta Leonor Thrombosis Research Group
}

Published online: 29 June 2020

(c) Springer Science+Business Media, LLC, part of Springer Nature 2020

\begin{abstract}
Recent studies suggest that thrombotic complications are a common phenomenon in the novel SARS-CoV-2 infection. The main objective of our study is to assess cumulative incidence of pulmonary embolism (PE) in non critically ill COVID-19 patients and to identify its predicting factors associated to the diagnosis of pulmonary embolism. We retrospectevely reviewed 452 electronic medical records of patients admitted to Internal Medicine Department of a secondary hospital in Madrid during Covid 19 pandemic outbreak. We included 91 patients who underwent a multidetector Computed Tomography pulmonary angiography(CTPA) during conventional hospitalization. The cumulative incidence of PE was assessed ant the clinical, analytical and radiological characteristics were compared between patients with and without PE. PE incidence was $6.4 \%$ (29/452 patients). Most patients with a confirmed diagnosed with PE recieved low molecular weight heparin (LMWH): 79.3\% (23/29). D-dimer peak was significatly elevated in PE vs non PE patients $(14,480 \mathrm{vs} 7230 \mathrm{mcg} / \mathrm{dL}, \mathrm{p}=0.03)$. In multivariate analysis of patients who underwent a CTPA we found that plasma D-dimer peak was an independen predictor of PE with a best cut off point of $>5000 \mu \mathrm{g} / \mathrm{dl}$ (OR 3.77; IC95\% (1.18-12.16), $\mathrm{p}=0.03$ ). We found ninefold increased risk of PE patients not suffering from dyslipidemia (OR 9.06; IC95\% (1.88-43.60). Predictive value of AUC for ROC is 75.5\%. We found a high incidence of PE in non critically ill hospitalized COVID 19 patients despite standard thromboprophylaxis. An increase in D-dimer levels is an independent predictor for PE, with a best cut-off point of $>5000 \mu \mathrm{g} / \mathrm{dl}$.
\end{abstract}

Keywords COVID-19 $\cdot$ Pulmonary embolism $\cdot$ Hypercoagulable state $\cdot$ Antithrombotic therapy $\cdot$ Computed tomography pulmonary angiography

\section{Highlights}

The members of the Infanta Leonor Thrombosis Research Group have been listed in acknowledgements.

N. Muñoz-Rivas

nmunozr@salud.madrid.org

1 Internal Medicine Department, Infanta Leonor University Hospital, Gran Via del Este Avenue, 80., 28031 Madrid, Spain

2 Radiology Department, Infanta Leonor University Hospital, Madrid, Spain

3 Medical Oncology Department, Infanta Leonor University Hospital, Madrid, Spain

4 Anesthesiology Department, Infanta Leonor University Hospital, Madrid, Spain
- High incidence of PE (6.4\%) despite thromboprophylaxis in patients admitted in general ward with COVID 19.

5 Cardiology Department, Infanta Leonor University Hospital, Madrid, Spain

6 Department of Hospital Pharmacy, Infanta Leonor University Hospital, Madrid, Spain

7 Nursing Department, Infanta Leonor University Hospital, Madrid, Spain

8 Hematoloy Department, Infanta Leonor University Hospital, Madrid, Spain

9 Universidad Complutense de Madrid, Madrid, Spain 
- An increase in D-Dimer levels with a best cut -off point of $>5000 \mu \mathrm{g} / \mathrm{dl}$ is an independent predictor of PE.

- There was no difference in mortality between patients with COVID-19 with vs without PE in our cohort of non critically ill COVID 19 patients.

- Dyslipidemia was a protective factor in our study, ongoing trials with statins will offer evidence in this area.

\section{Introduction}

The novel coronavirus called SARS-CoV-2 emerged in the city of Wuhan, China, on December 31st, 2019. The infection by SARS-CoV-2 (COVID-19) affects mainly the respiratory system with a variable clinical presentation, from a flu-like syndrome with mild respiratory symptoms to a viral pneumonia with acute respiratory distress syndrome, multiple organ dysfunction and death. Recent observational studies reported an increased incidence of thrombotic complications including pulmonary embolism ( $\mathrm{PE}$ ) and venous thromboembolism (VTE) [1, 2]. In fact, autopsy reports from SARS-CoV-1 and MERS infected patients had shown lung microvascular thrombosis [3]. Preliminary reports on COVID-19 patients' coagulation disorders showed thrombocytopenia, elevated D-dimer, prolonged prothrombin time, and disseminated intravascular coagulation. These coagulation alterations have been associated with poor prognosis [4]. Multiple pathogenic mechanisms are involved, including activation of macrophages, monocytes, platelets and lymphocytes, endothelial dysfunction, Toll-like receptor and tissue-factor pathway activation. This syndrome is called MicroCLOTS (microvascular COVID-19 lung vessels obstructive thromboinflammatory syndrome) [5]. The aim of this study is to assess the cumulative incidence of pulmonary embolism (PE) in non critically ill COVID-19 patients and associated predicting factors.

\section{Material and methods}

\section{Study design}

Single cohort, longitudinal study of patients admitted with COVID-19 diagnosis to the Internal Medicine Department of Infanta Leonor University Hospital in Madrid between March 30th, 2020 and April 12th, 2020. We retrospectively reviewed 452 medical records of all consecutive admitted patients. Among these patients, we selected those who underwent a CTPA during their hospital admission. We analyzed cumulative incidence of PE in COVID-19 diagnosed patients and associated risk factors. COVID-19 diagnosis was based on WHO clinical criteria [6] and/or confirmed by RT-PCR of nasopharyngeal specimens. The medical criteria for indication of CT scans during hospital admission to rule out PE were respiratory deterioration not attributable to other causes, data on acute respiratory distress without improvement despite specific treatment or elevation of D-dimer in discordance with other inflammatory parameters.

Approval was obtained from local ethics committee of the Infanta Leonor University Hospital, the study belongs to the COVID -19@Vallecas cohort. This study complied with the declaration of Helsinki and was approved by the local ethics and investigation committee. Written informed consent was waived due to the retrospective nature of the study. Collected data were appropriately made anonymous, and each patient was identified by a unique alphanumeric identification code.

\section{CT protocol}

Our routine protocol for CTPA in patients with clinical suspicion of PE was performed in a 40 row CT (Somatom Sensation, Siemens Healthineers, Erlangen, Germany) after intravenous injection of $70-80 \mathrm{ml}$ of iodinated contrast agent (Iohexol $300 \mathrm{mg} / \mathrm{ml}$, Omnipaque 300, GE Healthcare Biosciences) at a flow rate of $4 \mathrm{~mL} / \mathrm{s}$, with the use of a bolustracking technique and a threshold of $100 \mathrm{HU}$ triggered on the main pulmonary artery. Reformatted images were obtained with a slice-thickness of $0.75 \mathrm{~mm}$ in mediastinal and parenchymal windows. The most proximal luminal defect within pulmonary vessels was employed to define PE location as main pulmonary arteries, lobar, segmental or subsegmental thromboembolism.

\section{Statistical analysis}

Patients were divided into two subgroups depending on a positive or negative diagnosis of PE in the CTPA. A descriptive analysis of the characteristics of both groups was performed: for the categorical variables Pearson chi square test or Fisher exact test was performed. For continuous variables with a normal distribution means and standard deviations (SD) are shown, for variables that do not have a normal distribution median and interquartile range (IQR) were used. Student T test or Wilcoxon rank tests were performed according to whether they followed a normal distribution or not. Normal distribution was checked visually by a histogram, and numerically by the Saphiro-Wilktest. A multivariate logistic regression was performed to predict the independent contribution of different factors in PE diagnosed patients and in those without PE. In the multivariate model, cut points that maximize the separation of the target variable by using Segmentation Trees models were introduced to avoid logarithmic transformations that could make interpretation difficult in variables that do not have a normal distribution. The $p$ values reported correspond to the 2 -tailed analysis. A p-value $<0.05$ was considered statistically 
significant. All statistical analysis was performed using the statistical package SAS 9.4 (Copyright $\odot 2016$ by SAS Institute Inc., Cary, NC, USA.

\section{Results}

We analyzed 452 electronic medical records. $91 \mathrm{CT}$ scans were performed, 29 patients (31.9\%) were diagnosed with acute PE, with a cumulative incidence of $6.4 \%$ (29/452 patients).

\section{Patients with pulmonary embolism}

All of these patients were classified as COVID-19 infection (23 patients by RT-PCR positive and 6 with positive CT scan and negative RT-PCR). In PE group, 72.0\% (21/29) were male and the median age was 65 years (IQ 1-3: 56-73). The median body mass index (BMI) was $28.8 \mathrm{~kg} /$ $\mathrm{m}^{2}$ (IQ 1-3:26.8-31.8). Among their comorbidities 41.3\% (12/29) had arterial hypertension, $47.0 \%$ (8/17) were obese $\left(\mathrm{BMI}>30 \mathrm{~kg} / \mathrm{m}^{2}\right), 10.3 \%$ (3/29) had diabetes mellitus, $10,3 \%$ (3/29) had dyslipidemia and $17.2 \%(5 / 29)$ had a medical history of chronic obstructive pulmonary disease (Table 1). In the analytical variables, median plasma D-dimer peak was $14,480 \mu \mathrm{g} / \mathrm{dl}$ (IQ 1-3: 5540-33,170 $\mu \mathrm{g} / \mathrm{dl}$ ), median platelet counts was $137 \times 10^{3}$ (IQ 1-3: $248-260 \times 10^{3}$ ), median C-reactive protein $110.6 \mathrm{mg} / \mathrm{dl}$ (Q1-3: 40-193) and median ferritin $829 \mathrm{ng} / \mathrm{ml}$ (Q1-3: 387-1272). There was no associated coagulopathy, with a prothrombin time of $12.5 \mathrm{~s}(\mathrm{Q} 1-$ Q3: 11.9-13.5). Regarding radiological findings, a 51.7\% of PE were bilateral (15/29 patients) and $48.3 \%$ unilateral. Most PE were in a peripheral location in segmental and subsegmental arteries (68.9\%, 20/29 patients) and 31.0\% (9/29 patients) in a central (main and lobar arteries) (Fig. 1).

It should be noted that up to $79.3 \%$ (23/29) of the patients were under treatment with LMWH at prophylactic doses at the time of diagnosis of PE.

\section{PE versus non PE patients}

We compared predictive known risk factors of thrombosis between both groups (Table 2).

Statistically significant differences were found between the two groups for history of dyslipidemia, $10.7 \%,(3 / 29)$ in PE patients versus $40.3 \%(25 / 62), \mathrm{p}=0.003)$ and a history of autoimmune disease (3/29 in PE patients versus $0 / 620 \%$, $\mathrm{p}=0.03$ ). No statistically significant differences were found in BMI, history of previous thrombosis (PE or deep vein thrombosis [DVT]), personal history of thrombophilia, solid or hematological malignancy or history of lung disease.
Table 1 Clinical and radiological characteristics in PE patients

\begin{tabular}{|c|c|}
\hline Variable & Results $(\mathrm{N}=29)$ \\
\hline Age, median (Q1-Q3) & $65(56-73)$ \\
\hline Men & $21 / 29(72 \%)$ \\
\hline Women & $8 / 29(28 \%)$ \\
\hline $\begin{array}{l}\text { Days from COVID19 symptoms to PE diagnosis, } \\
\text { median (Q1-Q3) }\end{array}$ & $18(12.5-22)$ \\
\hline SARS-CoV2 nasopharyngeal RT-PCR & $23 / 29(79.3 \%)$ \\
\hline \multicolumn{2}{|l|}{ Clinical situation } \\
\hline Blood pressure, median (Q1-Q3) (mmHg) & $128.5(118-144)$ \\
\hline Heart rate (beats/min) median (Q1-Q3) & $95(84-103)$ \\
\hline Respiratory rate (breaths/min) median (Q1-Q3) & $18(16-22)$ \\
\hline Blood oxygen saturation (\%) median (Q1-Q3) & $94(91-98)$ \\
\hline Basal & $3 / 28(10.7 \%)$ \\
\hline Nasal cannula & $10 / 28(35.7 \%)$ \\
\hline High flow oxygen mask & $7 / 28(25.0 \%)$ \\
\hline NIMV & $8 / 28(28.6 \%)$ \\
\hline Temperature $\left({ }^{\circ} \mathrm{C}\right)$ median $(\mathrm{Q} 1-\mathrm{Q} 3)$ & $37.2(36.5-38.1)$ \\
\hline \multicolumn{2}{|l|}{ PE extent } \\
\hline Bilateral & $15 / 29(51.7 \%)$ \\
\hline Unilateral & $14 / 29(48.3 \%)$ \\
\hline \multicolumn{2}{|l|}{ PE location } \\
\hline Central & $9 / 29(31.0 \%)$ \\
\hline Peripheral & $20 / 29(68.9 \%)$ \\
\hline DVT & $2 / 29(6.9 \%)$ \\
\hline \multicolumn{2}{|l|}{ Therapy at PE diagnosis } \\
\hline LMH prophylactic doses & $23 / 29(79.3 \%)$ \\
\hline Antibiotics & $27 / 28(96.4 \%)$ \\
\hline Hidroxicloroquine & $28 / 29(96.5 \%)$ \\
\hline Sterois (IV) & $18 / 29(62.1 \%)$ \\
\hline Tocilizumab & $22 / 26(84.6 \%)$ \\
\hline \multicolumn{2}{|l|}{ Outcomes } \\
\hline Discharged & $24 / 29(82.7 \%)$ \\
\hline Remains at hospital & $4 / 29(13.8 \%)$ \\
\hline ICU & $2 / 29(6.9 \%)$ \\
\hline Death & $1 / 29(3.4 \%)$ \\
\hline
\end{tabular}

$N I M V$ noninvasive mechanical ventilation, $I C U$ intensive care unit

Patients with PE had higher D-dimer levels (median 14,480 $\mu \mathrm{g} / \mathrm{dl}$, IQR 5540-33,170) compared to patients without PE $(7230 \mu \mathrm{g} / \mathrm{dl}$, IQR $2100-16,415$, p value $=0.03)$.

No differences were found in platelets count, fibrinogen levels, renal function, ferritin, PCR levels, IL-6 and liver function.

In our results, patients treated with $\mathrm{ARB}$ are less likely to have PE (0/29 in PE patients versus 8/62, Fisher`s exact test $p=0.05)$. No differences were found in other treatments during admission.

In our study population we did not find statistical differences for admission to intensive care unit and mortality between patients with and without PE. 


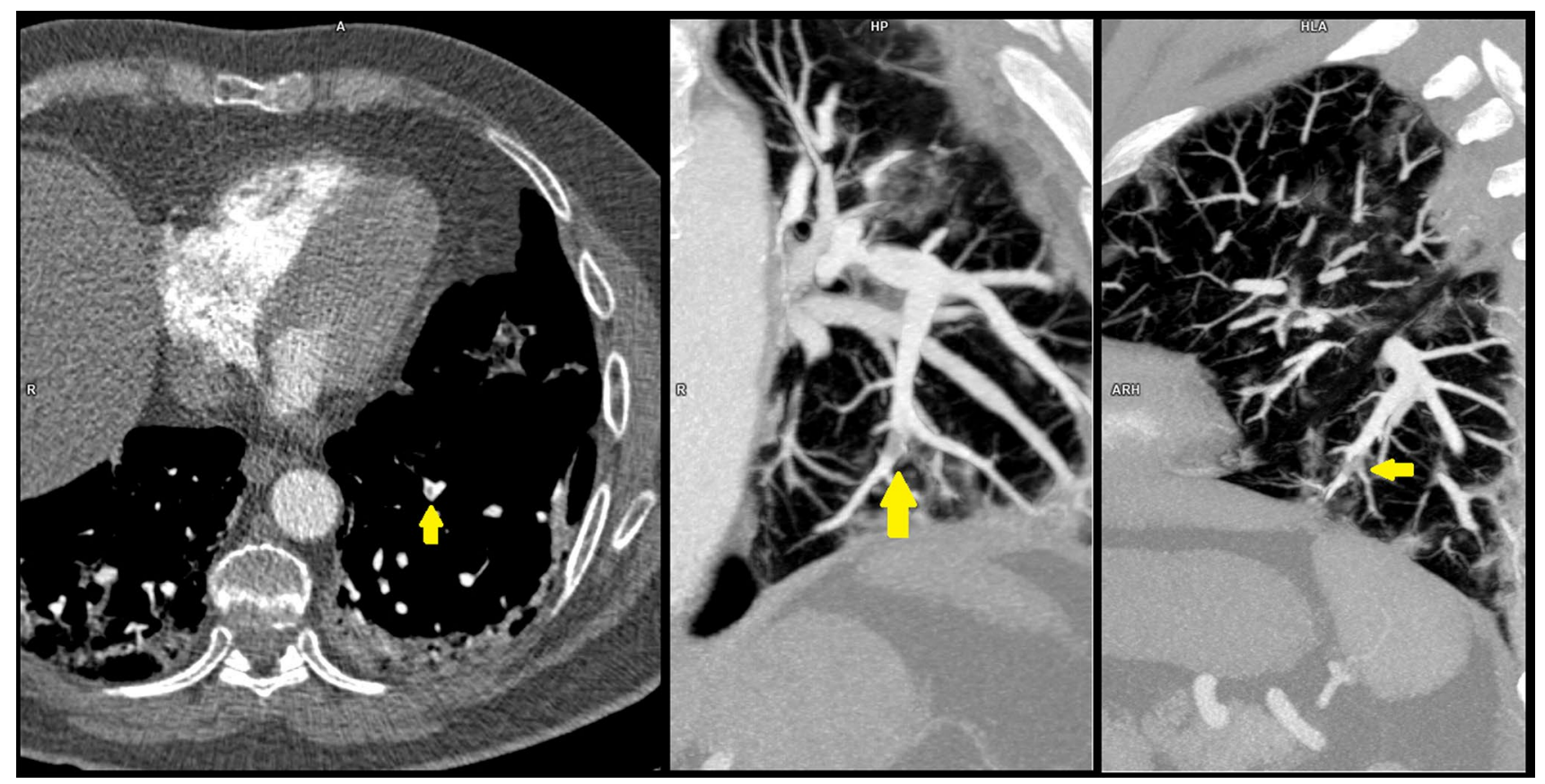

Fig. 1 Axial CT angiography scan and reformatted images show a pulmonary embolus within a segmental left lower lobe artery (arrows) that results in a partial filling defect, which is surrounded by contrast material

Factors associated with PE by univariate analysis were history of dyslipidemia, history of autoimmune disease, D-dimer levels and treatment with ARB. By multivariate analysis, only history of dyslipidemia and D-dimer levels remained independently associated to PE. In multivariate logistic regression analysis we found that d-dimer level was an independent predictor of PE with a best cut off of $>5000 \mu \mathrm{g} / \mathrm{dl}($ OR 3.77; IC95\% $(1.18-12.16)(\mathrm{p}=0,03)$ and also the history of dyslipidemia. with a ninefold increased risk of PE patients not suffering from dyslipidemia (OR 9.06; IC95\% (1.88-43.60). Predictive value of the model including both variables (DD peak and history of dyslipidemia) (AUC for ROC is 75.5\%) is shown in Fig. 2.

\section{Discussion}

COVID-19 may predispose patients to thrombotic complications due to excessive inflammation, platelet activation, endothelitis and stasis [7]. Studies reporting both arterial and venous thrombotic events in patients with COVID-19 have emerged in the literature, especially among those who are severely and critically ill [8]. At present, data regarding thromboembolic risk and SARS-CoV-2 infection is still scarce and mainly coming from patients admitted to critical care units $[9,10]$. However, to the best of our knowledge, risk of pulmonary embolism in non critically ill patients has been less covered.
Our observational study provides information about cumulative incidence of PE in patients hospitalized with COVID-19 pneumonia in general ward of an Internal Medicine department in a secondary university hospital in Madrid. We found 29 patients with proven PE and COVID19 pneumonia out of 91 CTPA performed among the 452 patients admitted in the study period. That represents an incidence of $6.4 \%$ in a medical ward and one third of positive CTPA despite prophylactic doses of LWMH. Our findings are similar to those reported recently by Lodigiani et al. [10]. The authors reported 20 events occurred in patients on the general ward in an academic hospital in Milan (6.4\%) of a total of 388 patients included. These values appear much higher than the rate of symptomatic VTE events observed in thromboprophylaxis trials, not exceeding $1 \%$ in patients receiving thromboprophylaxis [11]. Not surprisingly, incidence of VTE in general wards is lower than observed in critically ill patients. Helms et al. in multicenter prospective cohort study of 150 ICU patients described a PE incidence of $16.7 \%$ also despite prophylactic or therapeutic anticoagulant treatment [12]. However, our figures could be understimated, we postulate this based on an active search of VTE performed in around 20\% of COVID-19 patients which demonstrated one third of positive VTE imaging tests. This rates are the same reported by Lorant et al. in their study of Strasbourg University Hospital [13] where they found thirtytwo of 106 (30\%, [CI95\% 22-40\%]) patients with COVID19 and with pulmonary CT angiogram positive for acute pulmonary embolism. 
Table 2 Baseline characteristics CT scan without and with PE

\begin{tabular}{|c|c|c|c|}
\hline $\mathrm{n}=91$ & CT scan without PE $n=62$ ) & CT scan with PE $(n=29)$ & $\mathrm{p}$ value \\
\hline Age (years) median (Q1-Q3) & $64.5(57-75)$ & $65(56-73)$ & $\mathrm{p}=0.5479$ \\
\hline Men & $41 / 6266 \%$ & $21 / 2972 \%$ & $\mathrm{p}=0.5488$ \\
\hline BMI, median $\left(\mathrm{kg} / \mathrm{m}^{2}\right)$ & 29.83 & 28.82 & $\mathrm{p}=0.8405$ \\
\hline$<25$ & $8 / 46(17 \%)$ & $1 / 17(5.8 \%)$ & \\
\hline $25-30$ & $15 / 46(32 \%)$ & $8 / 17(47 \%)$ & \\
\hline$>30$ & $23 / 46(50 \%)$ & $8 / 17(47 \%)$ & \\
\hline \multicolumn{4}{|l|}{ Comorbidities } \\
\hline Diabetes mellitus & $13 / 62(20.9 \%)$ & $3 / 29(10.3 \%)$ & $\mathrm{p}=0.2148$ \\
\hline Arterial hypertension & $35 / 62(56.4 \%)$ & $12 / 29(41.3 \%)$ & $\mathrm{p}=0,18$ \\
\hline Dyslipemia & $25 / 62(40,3 \%)$ & $3 / 29(10.3 \%)$ & $\mathrm{p}=0.0039$ \\
\hline Smoking & $5 / 62(8 \%)$ & $2 / 29(6.8 \%)$ & $\mathrm{p}=1.0$ \\
\hline Heart failure & $1 / 62(1.6 \%)$ & $1 / 29(3.4 \%)$ & $\mathrm{p}=0,5382$ \\
\hline Chronic obstructive lung disease & $8 / 62(12.9 \%)$ & $5 / 29(17.2 \%)$ & $\mathrm{p}=0.7487$ \\
\hline Asthma & $5 / 62(8 \%)$ & $2 / 29(6.8 \%)$ & $\mathrm{p}=1.0$ \\
\hline Apnea syndrome & $7 / 62(11.2 \%)$ & $3 / 29(10.3 \%)$ & $\mathrm{p}=1.0$ \\
\hline CPAP & $6 / 62(9.6 \%)$ & $2 / 29(6.8 \%)$ & $\mathrm{p}=1.0$ \\
\hline Chronic renal dysfuntion & $1 / 62(1.6 \%)$ & $1 / 29(3.4 \%)$ & $\mathrm{p}=0.5382$ \\
\hline Cirrosis & $0 / 62(0 \%)$ & $1 / 29(3.4 \%)$ & $\mathrm{p}=0.3187$ \\
\hline Prior stroke & $5 / 62(8 \%)$ & $1 / 29(3.4 \%)$ & $\mathrm{p}=0.6602$ \\
\hline Prior throembolic events & $2 / 62(2.8 \%)$ & $1 / 29(3.4 \%)$ & $\mathrm{p}=1.0$ \\
\hline Prior PE & $1 / 62(1.6 \%)$ & $1 / 29(3.4 \%)$ & $\mathrm{p}=0.5382$ \\
\hline Prior deep vein thrombosis & $1 / 62(1.6 \%)$ & $0 / 29(0 \%)$ & $\mathrm{p}=1.0$ \\
\hline Thrombophilia & $0 / 62(0 \%)$ & $0 / 62(0 \%)$ & \\
\hline \multicolumn{4}{|l|}{ Active cancer } \\
\hline Solid & $1 / 62(1.6 \%)$ & $2 / 29(6.8 \%)$ & $\mathrm{p}=0.2373$ \\
\hline Hematological & $0 / 62(0 \%)$ & $0 / 29(0 \%)$ & \\
\hline Autoimmune disease & $0 / 620 \%$ & $3 / 2910.3 \%$ & $\mathrm{p}=0.0301$ \\
\hline $\begin{array}{l}\text { Systolic blood pressure previous CT Scan, median } \\
\text { (Q1-Q3) (mmHG) }\end{array}$ & $130(112-145)$ & $128,5(118-144)$ & $\mathrm{p}=0.7527$ \\
\hline Peak D-dimer, median (Q1-Q3) ( $\mu \mathrm{g} / \mathrm{dl})$ & $7230(2105-16,415)$ & $14,480(5540-33,170)$ & $\mathrm{p}=0.0393$ \\
\hline Platelets, median (Q1-Q3) (x10E3) & $245(181-318)$ & $244(174-325)$ & $\mathrm{p}=0.84$ \\
\hline Fibrinogen, median (Q1-Q3) (mg/dl) & $269.5(205.5-431.5)$ & $181(160-321)$ & $\mathrm{p}=0.1247$ \\
\hline PT, median (Q1-Q3) (sg) & $12.45(11.8-13.3)$ & $12.5(11,9-13,5)$ & $\mathrm{p}=0.8905$ \\
\hline Creatinine, median (Q1-Q3) (mg/dl) & $1.04(0.9-1.2)$ & $1.06(1-1,2)$ & $\mathrm{p}=0.4518$ \\
\hline Ferritin, median (Q1-Q3) (ng/dl) & $826(246-2322)$ & $29(387-1272)$ & $\mathrm{p}=0.9202$ \\
\hline PCR, median (Q1-Q3) (mg/dl) & $126(48.4-207.5)$ & $110.6(40-193.1)$ & $\mathrm{p}=0.7711$ \\
\hline LDH, median (Q1-Q3) (U/1) & $322(237-435)$ & $372.5(227-433)$ & $\mathrm{p}=0.7914$ \\
\hline IL-6, median (Q1-Q3) (UI) & $50.85(21-105)$ & 64.1 & $\mathrm{p}=0.7630$ \\
\hline \multicolumn{4}{|l|}{ Treatment with: } \\
\hline NSAIDS & $5 / 62(8 \%)$ & $1 / 29(3.4 \%)$ & $\mathrm{p}=0.6602$ \\
\hline ACE inhibitors & $17 / 62(27.4 \%)$ & $10 / 29(34.4 \%)$ & $\mathrm{p}=0.4929$ \\
\hline ARBS & $8 / 62(12.9 \%)$ & $0 / 29(0 \%)$ & $\mathrm{p}=0,0515$ \\
\hline Inhaled steroids & $8 / 62(12.9 \%)$ & $2 / 29(6.8 \%)$ & $\mathrm{p}=0.4932$ \\
\hline Statins & $21 / 62(33.8 \%)$ & $6 / 29(20.6 \%)$ & $\mathrm{p}=0.1996$ \\
\hline Non-invasive mechanical ventilation & $23 / 61(37 \%)$ & $12 / 25(48 \%)$ & $\mathrm{p}=0-3775$ \\
\hline Exitus & $7 / 58(12 \%)$ & $1 / 29(3.4 \%)$ & $\mathrm{p}=0.2598$ \\
\hline
\end{tabular}

CPAP continous positive air pressure, IL-6 Interleukin-6, NSAIDS Nonsteroid anti-inflamatory drugs, ACE inhibitors angyotensin-converting inhibitors, $A R B s$ Angiotensin II receptor blockers 


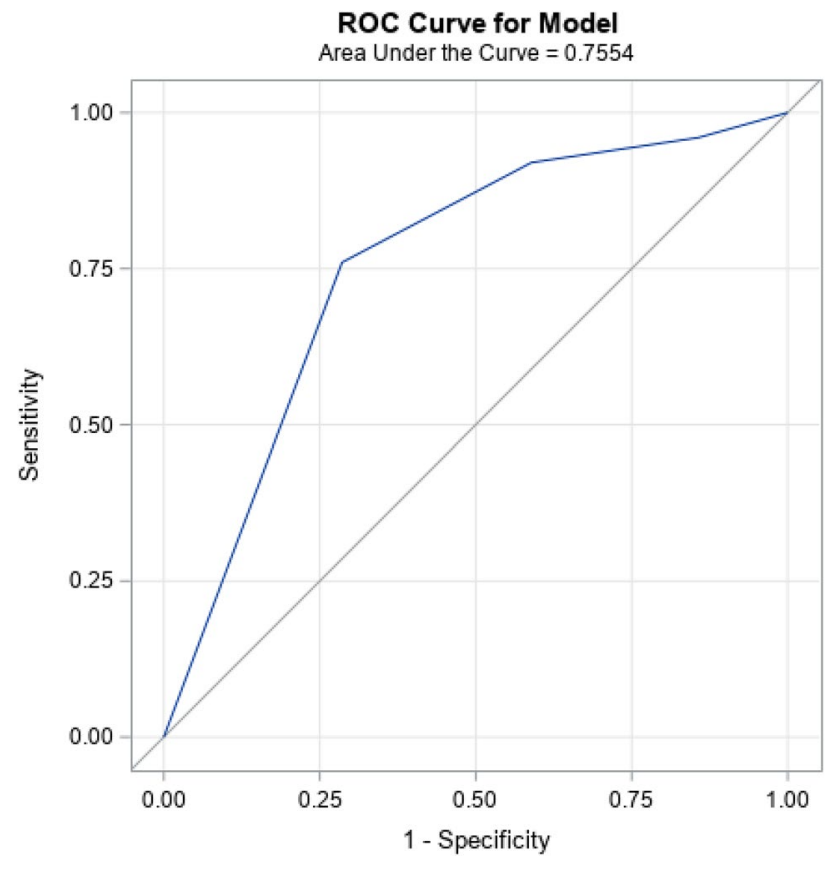

Fig. 2 ROC curve. AUC for the model $75.5 \%$

In our study the absence of classic risk factor for venous thromboembolism (advanced age, history of thrombosis, thrombophilia, cancer and ICU admission) and the peripherical localization of PE suggest microthrombosis in situ [5]. Wells index seems not to be accurate to predict PE in such a challenging context. Patients with COVID 19 pneumonia ill enough to be admitted to hospital share many clinical conditions, as immobilization, acute respiratory failure, inflammation status but also analytical parameters such as high D-dimers which make challenging the diagnosis of PE. All patients who underwent a CT scan during hospital admission to rule out PE had experimented respiratory deterioration not attributable to other causes or elevation of D-dimer in discordance with other inflammatory parameters.

Knowing risk factors for PE in COVID19 patients is crucial to establish the indication of CT lung angiography due to the logistical difficulty involved to practice this technique: mobilization of patients, acute respiratory failure, iatrogenic contrast, risk of transmission of the virus and optimization of resources in a situation of saturation care.

We found D-dimer peak levels significantly higher among patients with PE. D-dimer is a degradation product of cross-linked fibrin and reflects blood clot formation and its subsequent fibrinolysis. Several studies have shown a strong association between high D-dimer levels and poor prognosis in patients diagnosed with COVID -19 [14-16]. We founded a best cut-off of $>5000 \mu \mathrm{g} / \mathrm{dl}$ by segmentation tree, $83 \%$ of patients with PE had higher levels of DD. Unlike others, we did not found thrombocytopenia significantly associated to a positive diagnostic of PE, probably due to our small sample size. Tang et al. reported decreased platelet count as an independent risk factors for 28-day mortality [14]. Interestingly, we found the history of dislypidemia as a protector factor for PE in the multivariate analysis. Patients who did not have this cardiovascular risk in their medical records, had an ninefold increased risk for PE compared to those with dyslipidemia. A possible explanation could be that patients previously treated with statins had a potential benefit either by their immunomodulatory action or by preventing cardiovascular damage [17]. In our study we did not found significantly difference in treatment with statins while hospitalization in patients with and without TEP (20,6\% vs $35,4 \%, \mathrm{p}=0.15)$.

Among patients with COVID-19 undergoing CT to evaluate respiratory deterioration and/or elevated D-dimer concentration, the clinical characteristics of patients who did versus did not have PE identified were similar. We did not find statistical difference between both groups in comorbidities other than dsylipidemia, no more active cancer rates nor prior DVT or PE. Patients were mostly overweighted men, around 65 years old. There was no difference in severity of pneumonia by CURB-65 score, also non statistically difference in inflammation parameters (high in both groups), treatment or need of non invasive ventilation although figures are higher for non PE group. Importantly, the actual presence of PE on CT was not associated with mortality in this small sample. Bompard et al. recently reported that patients with PE were more frequently hospitalized in ICU and more frequently under mechanical ventilation but did not find differences in mortality rates in both groups [18]. In our series, PE were mostly (68\%) peripherical, subsegmental, probably in situ (incidence of concomitant DVT in these patients was only $6.8 \%$ ) and this is probably why PE has not conditioned an impact at the prognosis.

The main limitations of our study are its observational and rectrospective nature and small sample size. Another limitation of our study is the practically absence of screening of DVT in legs. Expert guidelines recommend universal thromboprophylaxis for patients admitted to hospital with suspected or confirmed COVID-19 pneumonia, and so does our local protocol irrespective of risk scores [7, 19].

In conclusion we found a high incidence of PE in non critically ill hospitalized COVID 19 patients despite standard thromboprophylaxis. Randomized controlled trials on VTE prevention will help to optimize therapeutic strategies. An increase in the D-dimer levels is a good predicting factor of PE, with best cut-off in $>5000 \mathrm{mcg} / \mathrm{dL}$.

Acknowledgements The authors thank Mrs Teresa de la Torre and Dr Ryan Murua. We also thank Professional Research Assistance, Mr Juan Francisco Dorado for statistical analysis and the Research Foundation of Infanta Leonor University Hospital. 
Infanta Leonor Thrombosis Research Group members: B. MestreGómez, R.M. Lorente-Ramos, J. Rogado, A. Franco-Moreno, B. Obispo, D. Salazar-Chiriboga, T. Saez-Vaquero, J. Torres-Macho, A. Abad-Moto, C. Cortina-Camarero, A. Such-Diaz, E. Ruiz-Velasco, N. Muñoz-Rivas, F. Sierra-Hidalgo, E. Moya-Mateo, M. de CarranzaLópez, M.A. Herrera-Moroueco, M. Akasbi-Montalvo, V. PardoGuimerá, P. Medrano-Izquierdo, E. Mariscal-Gómez, K. Marín-Mori, C. Figueras-González, S. López-Lallave, D. Díaz-Díaz, C. MauleónFernández, J. Martín-Navarro, P. Torres-Rubio, C. Matesanz, M.J. Moro-Alvarez, A. Bustamante-Fermosel, J.S.A Hernández-Rivas.

\section{Compliance with ethical standards}

Conflict of interest The authors have declared no conflicts of interest.

\section{References}

1. Driggin E, Madhavan MV, Bikdeli B et al (2020) Cardiovascular considerations for patients, health care workers, and health systems during the coronavirus disease 2019 (COVID-19) Pandemic. J Am Coll Cardiol. https://doi.org/10.1016/j.jacc.2020.03.031

2. Fried JA, Ramasubbu K, Bhatt R et al (2020) The variety of cardiovascular presentations of COVID-19. Circulation. https://doi. org/10.1161/CIRCULATIONAHA.120.047164

3. Giannis D, Ziogas IA, Gianni P (2020) Coagulation disorders in coronavirus infected patients: COVID-19, SARS-CoV-1, MERSCoV and lessons from the past. J Clin Virol 127:104362. https:// doi.org/10.1016/j.jcv.2020.104362

4. Tang N, Li D, Wang X, Sun Z (2020) Abnormal coagulation parameters are associated with poor prognosis in patients with novel coronavirus pneumonia. J Thromb Haemost 18:844-847. https://doi.org/10.1111/jth.14768

5. Ciceri F, Beretta L, Scandroglio AM, et al (2020) Microvascular COVID-19 lung vessels obstructive thromboinflammatory syndrome (MicroCLOTS): an atypical acute respiratory distress syndrome working hypothesis. Crit Care Resusc

6. WHO (2020) Coronavirus disease 2019 (COVID-19) Situation Report-94. https://www.who.int/docs/default-source/coron aviruse/situation-reports/20200423-sitrep-94-covid-19.pdf?sfvrs $\mathrm{n}=\mathrm{b} 8304 \mathrm{bf0} \_4$. Accessed 8 May 2020

7. Bikdeli B, Madhavan MV, Jimenez D et al (2020) COVID-19 and thrombotic or thromboembolic disease: implications for prevention, antithrombotic therapy, and follow-up. J Am Coll Cardiol. https://doi.org/10.1016/j.jacc.2020.04.031

8. Cui S, Chen S, Li X et al (2020) Prevalence of venous thromboembolism in patients with severe novel coronavirus pneumonia. J Thromb Haemost. https://doi.org/10.1111/jth.14830
9. Klok FA, Kruip MJHA, van der Meer NJM et al (2020) Incidence of thrombotic complications in critically ill ICU patients with COVID-19. Thromb Res. https://doi.org/10.1016/j.throm res.2020.04.013

10. Lodigiani C, Iapichino G, Carenzo L et al (2020) Venous and arterial thromboembolic complications in COVID-19 patients admitted to an academic hospital in Milan, Italy. Thromb Res 191:9-14. https://doi.org/10.1016/j.thromres.2020.04.024

11. Samama MM, Cohen AT, Darmon JY et al (1999) A comparison of enoxaparin with placebo for the prevention of venous thromboembolism in acutely ill medical patients. Prophylaxis in medical patients with Enoxaparin Study Group. N Engl J Med 341:793800. https://doi.org/10.1056/NEJM199909093411103

12. CRICS TRIGGERSEP Group (Clinical Research in Intensive Care, and Sepsis Trial Group for Global Evaluation, and Research in Sepsis), Helms J, Tacquard C et al (2020) High risk of thrombosis in patients with severe SARS-CoV-2 infection: a multicenter prospective cohort study. Intensive Care Med. https://doi. org/10.1007/s00134-020-06062-x

13. Leonard-Lorant I, Delabranche X, Severac F et al (2020) Acute pulmonary embolism in COVID-19 patients on CT angiography and relationship to D-Dimer levels. Radiology. https://doi. org/10.1148/radiol.2020201561

14. Tang N, Bai H, Chen X et al (2020) Anticoagulant treatment is associated with decreased mortality in severe coronavirus disease 2019 patients with coagulopathy. J Thromb Haemost 18:10941099. https://doi.org/10.1111/jth.14817

15. Zhou F, Yu T, Du R et al (2020) Clinical course and risk factors for mortality of adult inpatients with COVID-19 in Wuhan, China: a retrospective cohort study. Lancet 395:1054-1062. https://doi. org/10.1016/S0140-6736(20)30566-3

16. Chen J, Wang X, Zhang S et al (2020) Findings of acute pulmonary embolism in COVID-19 patients. Elsevier SSRN, Rochster

17. Castiglione V, Chiriacò M, Emdin M, Taddei S, Vergaro G (2020) Statin therapy in COVID-19 infection. Eur Heart J Cardiovasc Pharmacother. https://doi.org/10.1093/ehjcvp/pvaa042

18. Bompard F, Monnier H, Saab I et al (2020) Pulmonary embolism in patients with Covid-19 pneumonia. Eur Respir J. https://doi. org/10.1183/13993003.01365-2020

19. Oudkerk M, Büller HR, Kuijpers D et al (2020) Diagnosis, prevention, and treatment of thromboembolic complications in COVID19: report of the National Institute for Public Health of the Netherlands. Radiology. https://doi.org/10.1148/radiol.2020201629

Publisher's Note Springer Nature remains neutral with regard to jurisdictional claims in published maps and institutional affiliations. 